\title{
CRESCIMENTO E CONTEÚDO DE NUTRIENTES MINERAIS EM MUDAS DE MIRTILEIRO EM SISTEMA CONVENCIONAL E SEMI-HIDROPÔNICO ${ }^{1}$
}

\author{
DANIELE CAMARGO NASCIMENTO², MÁRCIA WULFF SCHUCH ${ }^{3}$, \\ ROBERTA MARINS NOGUEIRA PEIL ${ }^{4}$
}

RESUMO - O mirtileiro possui alto potencial econômico, no entanto, sua expansão de cultivo é limitada pela dificuldade de propagação. O cultivo hidropônico possibilita melhor crescimento devido à eficiência no fornecimento de nutrientes. O objetivo deste estudo foi avaliar o crescimento e o conteúdo de nutrientes minerais de mudas micropropagadas de mirtileiro em sistema convencional e semi-hidropônico. Mudas das cultivares Bluebelle e Woodard, com 3 meses de aclimatização, foram submetidas a dois sistemas de cultivo: sistema convencional,em que foram mantidas em sacos de polietileno contendo Plantmax ${ }^{\circledR}+$ vermiculita (1:1) e irrigadas com solução nutritiva a cada 15 dias; e sistema semi-hidropônico, na qual permaneceram em floreiras plásticas contendo areia, sendo irrigadas com solução nutritiva diariamente. O delineamento experimental foi em blocos casualizados com quatro tratamentos, com quatro repetições compostas por doze plantas. Foram avaliados mensalmente a altura da parte aérea, número de brotações e comprimento médio das brotações, e aos 90 dias a massa fresca da parte aérea, massa fresca e seca radicular e análise nutricional das folhas. O sistema semi-hidropônico proporcionou melhores resultados para todas as variáveis avaliadas, em ambas as cultivares. As mudas cultivadas no sistema semi-hidropônico apresentaram teores de nutrientes mais elevados e adequados para esta cultura.

Termos para indexação: propagação, microestaquia, hidroponia, Vaccinium spp.

\section{GROWTH AND MINERAL NUTRIENT CONTENT OF BLUEBERRY TRANSPLANTS IN CONVENTIONAL AND SEMI-HYDROPONIC SYSTEMS}

\begin{abstract}
The blueberry has high economic potential, but the, expansion of cultivation is limited by the difficulty of propagation. The hydroponic system provides a better growth due to the efficiency of nutrient supply. The objective of this study was to evaluate the growth and mineral nutrient content of blueberry transplants in conventional and semi-hydroponic system. Micropropagated transplants of Bluebelle and Woodard cultivars with three months of acclimatization were submitted to two cultivation systems: conventional system, where they were kept in polyethylene sacs containing Plantmax ${ }^{\circledR}+$ vermiculite $(1: 1)$ and irrigated with nutrient solution every 15 days; and semi-hydroponic, where they were kept in plastic flower pots containing sand and irrigated daily with nutrient solution. The experimental design was randomized blocks with four treatments, with four repetitions composed of twelve plants. Shoot height, number of shoots, average length of shoots, shoot fresh weight, fresh and dry mass of roots, and leaf nutritional analysis were evaluated. The semi-hydroponic system provided better results for all variables evaluated in both cultivars. Plants grown in semi-hydroponic system presented higher levels of nutrients which were appropriate for this culture.
\end{abstract}

Index terms: propagation, microcutting, hydroponics, Vaccinium spp.

\footnotetext{
${ }^{1}$ (Trabalho 065-11). Recebido em: 04-02-2011. Aceito para publicação em: 23-09-2011. A pesquisa é parte integrante da dissertação de mestrado do primeiro autor.

${ }^{2}$ Mestre do Programa de Pós-Graduação em Agronomia, Área de Concentração em Fruticultura de Clima Temperado, Universidade Federal de Pelotas (UFPel), Pelotas-RS. E-mail: den.biologia@gmail.com

${ }^{3}$ Eng. Agr. Dra ${ }^{\mathrm{a}}$, Laboratório de Micropropagação de Plantas Frutíferas, Departamento de Fitotecnia, Universidade Federal de Pelotas (UFPel), Pelotas-RS. E-mail: marciaws@ufpel.tche.br

${ }^{4}$ Eng. Agr. Dra., Departamento de Fitotecnia, Universidade Federal de Pelotas (UFPel), Pelotas-RS. E-mail: rmpeil@ufpel.edu.br
} 


\section{INTRODUÇÃO}

O mirtileiro é uma planta arbustiva de pequeno porte pertencente à família Ericaceae. É uma frutífera de clima temperado nativa dos Estados Unidos e Canadá. Essa cultura, até pouco tempo desconhecida para alguns produtores e consumidores, vem tornando-se cada vez mais popular.

No Sul do Brasil, esta espécie vem sendo pesquisada e produzida nova alternativa na área de fruticultura, pois apresenta alta rentabilidade, devido à baixa utilização de insumos e, até o momento, facilidade de produção limpa, resguardando o ambiente e a segurança alimentar (SANTOS; RASEIRA, 2002).

Do grupo das pequenas frutas que abrange, entre outras, as culturas de morango, framboesa, mirtilo e amora-preta, o mirtilo é classificado como a fruta fresca mais rica em antioxidante já estudada, tendo um conteúdo elevado de polifenóis tanto na casca quanto na polpa (PAYNE, 2005), podendo ser consumido in natura ou em produtos processados.

A expansão desta cultura é limitada pela dificuldade de propagação, indisponibilidade e qualidade das mudas. Segundo Ristow et al. (2009), além do baixo enraizamento, o mirtileiro apresenta lento desenvolvimento e baixo índice de sobrevivência das mudas após a formação das raízes.

A propagação do mirtilo pode ser realizada por sementes, enxertia, estaquia, miniestaquia e microestaquia. $\mathrm{O}$ sucesso na propagação vegetativa de espécies lenhosas está associado ao grau de juvenilidade da planta. A capacidade de rejuvenescimento que a micropropagação proporciona, pode ser utilizada na produção de mudas de mirtileiro inicialmente, utilizando-se a partir daí a microestaquia (Schuch, 2008). De acordo com Antunes (2007), as plantas obtidas por cultura de tecidos não devem ser levadas diretamente a campo, pois, além de novas, são muito tenras e sensíveis às variações ambientais.

No sistema convencional, as matrizes são selecionadas e acondicionadas em sacos de polietileno preto ou em vasos contendo substrato, formando um microjardim clonal em um espaçamento reduzido para a produção de estacas.

Estudos realizados com espécies florestais têm analisado o crescimento das mudas em sistemas hidropônicos para substituir o sistema convencional de cultivo. Esta evolução do microjardim clonal convencional ao sistema hidropônico reduziu problemas de propagação da cultura do eucalipto, como a sazonalidade e a produção de microestacas.

A hidroponia é uma técnica de cultivo protegido que utiliza solução nutritiva contendo os nutrientes necessários para o crescimento e o desen- volvimento das plantas, podendo ser associada com a utilização de substratos inertes.

Segundo Christofidis (2002), a disponibilidade de água tem gerado bastante discussão sobre a necessidade do emprego de técnicas agrícolas que reduzam sua utilização ou aumentem sua eficiência. Apesar de ser um fator fundamental no preparo da solução nutritiva, a água é usada de forma racional e eficiente no cultivo hidropônico. Além desta vantagem, a hidroponia possibilita a eficiência do uso de fertilizantes, a padronização da cultura, melhor controle do crescimento vegetativo, maior rendimento e qualidade da produção e independência em relação às condições do solo.

Entretanto, muitos cultivos hidropônicos não obtêm sucesso, principalmente devido ao desconhecimento dos aspectos nutricionais deste sistema de produção, isto é, à formulação e ao manejo mais adequado das soluções nutritivas (FURLANI, 2009).

O jardim clonal hidropônico pode ser estabelecido em diversos tipos de recipientes, como vasos ou floreiras de polipropileno, telhas de fibrocimento e canaletões de fibrocimento ou madeira, podendo ser auxiliado pela utilização de substratos inertes, como areia, brita e espuma fenólica.

Devido à diversidade de tipos de substratos que podem ser utilizados para a produção de mudas em hidroponia, tem aumentado a necessidade de estudos que avaliem o comportamento destes na produção de mudas.

Considerando a importância da propagação clonal de mirtileiro na fruticultura e a carência de estudos desta frutífera em relação à hidroponia, tornase justificável o desenvolvimento de pesquisas que envolvam sistemas semi-hidropônicos e hidropôni$\cos$, principalmente no que se refere à avaliação da sua eficiência na produção e manutenção de jardim microclonal.

Este trabalho tem como objetivo avaliar o crescimento e o conteúdo de nutrientes de mudas micropropagadas de mirtileiro para formação de microjardim clonal em sistema convencional e semihidropônico.

\section{MATERIAL E MÉTODOS}

O experimento foi conduzido no Campo Didático e Experimental do Departamento de Fitotecnia, no Câmpus da Universidade Federal de Pelotas, no Município de Capão do Leão-RS, durante os meses de outubro de 2009 a janeiro de 2010.

Foram utilizadas mudas micropropagadas de duas cultivares de mirtileiro, Bluebelle e Woodard. As mudas foram enraizadas em caixas plásticas, 
tamanho $22 \times 14 \times 10 \mathrm{~cm}$, contendo vermiculita e aclimatizadas em sacos de polietileno preto, tamanho de $10 \times 15 \mathrm{~cm}$, com substrato composto por Plantmax ${ }^{\circledR}+$ vermiculita $(1: 1)$, no volume de 250 $\mathrm{mL}$. O delineamento experimental foi o em blocos casualizados, com quatro tratamentos combinados às duas cultivares e aos dois sistemas de produção (convencional e hidropônico), cada um com quatro repetições, cada repetição composta por doze plantas $(1=$ Woodard + convencional; $2=$ Woodard + semihidropônico; $3=$ Bluebelle + convencional, e $4=$ Bluebelle + semi-hidropônico).

No sistema convencional, as mudas foram mantidas em sacos de polietileno preto, tamanho de $10 \times 15 \mathrm{~cm}$, com substrato composto por Plantmax ${ }^{\circledR}$ + vermiculita (1:1), em espaçamento de $10 \times 10 \mathrm{~cm}$. Neste sistema, a solução nutritiva era fornecida a cada 15 dias (aprox. $80 \mathrm{ml} /$ muda/dia).

No sistema semi-hidropônico, as mudas foram acondicionadas em floreiras plásticas, com 80 x $20 \times 25 \mathrm{~cm}$. No interior da floreira, foi colocada uma camada de $5 \mathrm{~cm}$ de brita para a drenagem e uma camada de $20 \mathrm{~cm}$ de areia grossa. As mudas de mirtileiro foram transplantadas para as floreiras, na proporção de doze mudas por floreira, distribuídas em duas linhas, no espaçamento de 10 x $10 \mathrm{~cm}$. As mudas eram irrigadas com solução nutritiva diariamente ( $1.000 \mathrm{ml} /$ floreira/dia). A solução nutritiva utilizada em ambos os sistemas foi formulada de acordo com as necessidades da cultura do mirtileiro.

A solução nutritiva utilizada em ambos os sistemas foi formulada de acordo com as necessidades da cultura do mirtileiro por Schuch e Peil (2011), com a seguinte composição de macronutrientes (em mmol litro $^{-1}$ ): 5,1 de $\mathrm{NO}_{3}^{-}, 1,1$ de $\mathrm{H}_{2} \mathrm{PO}_{4}^{-}, 3,7$ de $\mathrm{SO}_{4}^{2-}, 3,8$ de $\mathrm{NH}_{4}^{+}, 3,0$ de $\mathrm{K}^{+}, 2,3$ de $\mathrm{Ca}^{2+}$ e 1,1 de $\mathrm{Mg}^{2+}$; e de micronutrientes (em mg litro ${ }^{-1}$ ): 1,4 de Fe, 0,6 de Mn, 0,3 de Zn, 0,1 de Cu, 0,5 de B e 0,07 de Mo. Para o preparo da solução nutritiva, empregaram-se água da chuva e os seguintes fertilizantes e produtos: nitrato de cálcio, nitrato de amônio, fosfato monopotássico, sulfato de potássio, sulfato de magnésio, sulfato de amônio, ácido bórico, sulfato de cobre, sulfato de ferro, Na-EDTA, sulfato de manganês, sulfato de zinco e molibdato de sódio. $\mathrm{O}$ pH foi mantido entre 4,5 e 5,5 , e a condutividade elétrica (CE) foi de $1,4 \mathrm{dS} \mathrm{m}^{-1}$.

As avaliações das variáveis: altura da parte aérea, número de brotações e comprimento médio das brotações foram realizadas na instalação do experimento e aos 30; 60 e 90 dias. Na última avaliação, foram analisadas: a massa fresca da parte aérea, massa fresca e seca radicular e análise nutricional das folhas. Para a análise nutricional das folhas, as amostras de cada tratamento foram moídas e subme- tidas a digestões nítrico-perclórica e sulfúrica, sendo determinadas as concentrações dos elementos nos extratos obtidos, segundo a metodologia descrita por Tedesco et al. (1995). Para a interpretação dos teores médios dos macronutrientes e micronutrientes, foram utilizadas as recomendações de SOCIEDADE BRASILEIRA DE CIÊNCIA DO SOLO. COMISSÃO DE QUÍMICA E FERTILIDADE DO SOLO (2004) para os estados do Rio Grande do Sul e de Santa Catarina.

Os dados foram submetidos à análise da variância, pelo teste $\mathrm{F}$, e, quando significativos, submetidos à comparação entre médias, pelo teste de Tukey, a 5\% de significância. As médias foram analisadas por regressão polinomial, e o programa estatístico utilizado foi o WinStat, versão 2.0 (MACHADO ; CONCEIÇÃO, 2003).

\section{RESULTADOS E DISCUSSÃO}

Os resultados da análise de variância mostraram efeitos significativos da interação entre os fatores sistema $\mathrm{x}$ cultivar $\mathrm{x}$ tempo de cultivo para a altura da parte aérea e o número de brotações. Entre os fatores cultivar $\mathrm{x}$ tempo de cultivo para comprimento médio de brotações e, entre os fatores sistema $\mathrm{x}$ cultivar, para massa fresca e massa seca radicular.

Aos 60 dias de cultivo, pôde ser observada uma diferença significativa para altura da parte aérea e número de brotações, quando o sistema semihidropônico proporcionou melhores resultados para as duas cultivares testadas (Figura 1A e 1B).

Aos 30 dias de cultivo, as cultivares Woodard e Bluebelle diferenciaram-se entre si para comprimento médio de brotações $(6,47$ e 4,38 cm), quando a cultivar Woodard apresentou melhor resultado. No decorrer do tempo de avaliação, as cultivares passaram a ter um comportamento semelhante, não apresentando diferenças significativas em relação à esta variável (Figura 2).

Aos 90 dias de cultivo, verificou-se que o sistema semi-hidropônico proporcionou maior altura da parte aérea para as duas cultivares analisadas, Woodard e Bluebelle $(28,45$ e 29,79 cm), maior número de brotações $(6,47$ e 8,91$)$, maior massa fresca da parte aérea $(222,04$ e $233,53 \mathrm{~g})$, maior massa fresca radicular $(70,84$ e 30,14 g) e maior massa seca radicular (23,04 e 13,62 g) (Tabela 1). A cultivar Bluebelle não mostrou diferença significativa para massa fresca radicular. A cultivar Woodard foi inferior à Bluebelle para a variável número de brotações no sistema semi-hidropônico. A cultivar Bluebelle foi inferior à Woodard para a altura da parte aérea no sistema convencional e para massa fresca radicular e 
massa seca radicular no sistema semi-hidropônico.

Ristow et al. (2009), testando o crescimento de mudas micropropagadas de mirtileiro em diferentes substratos, verificaram $18 \mathrm{~cm}$ de comprimento da maior brotação com o substrato Plantmax ${ }^{\circledR}$ aos 90 dias de cultivo.

O fornecimento da solução nutritiva no sistema semi-hidropônico possibilitou uma alta disponibilidade dos nutrientes e, consequentemente, promoveu melhor crescimento vegetativo das mudas. Segundo Freire (2006), o máximo crescimento do mirtilo, tanto cultivado em areia, como em solução nutritiva, é obtido com o uso de cerca da metade da concentração de nutrientes usados para as demais frutíferas, sendo as plantas jovens mais sujeitas a graves danos causados pelo uso de doses excessivas de fertilizantes.

Souza (2007), comparando a produtividade de minicepas de Grevillea robusta nos sistemas de tubete e canaletão, concluiu que a produção de miniestacas no sistema em canaletão foi superior em relação às produzidas em tubete. $\mathrm{O}$ sistema em canaletão consiste em um sistema semi-hidropônico semelhante ao utilizado no presente trabalho, porém, ao invés de floreiras plásticas, foram utilizadas telhas de fibrocimento como canais de cultivo.

Cunha (2008) também obteve melhores resultados com o sistema hidropônico em relação ao sistema convencional, trabalhando com miniestaquia de corticeira-do-mato, obtendo maior altura, diâmentro do colo e produtividade de miniestacas.

Quanto à análise de nutrientes, observou-se interação entre os fatores sistema x cultivar para os teores de potássio e magnésio.

No sistema semi-hidropônico, observaramse maiores teores foliares de nitrogênio, fósforo, potássio, cálcio, magnésio e manganês para as duas cultivares testadas (Tabela 2). O mesmo sistema apresentou maior teor de cobre para a cultivar Woodard (Tabela 3). Os sistemas de cultivo não se diferenciaram para os teores de zinco e ferro. A cultivar Woodard apresentou maior teor de nitrogênio nos dois sistemas de cultivo, maior teor de zinco no sistema convencional e maior teor de manganês no sistema semi-hidropônico. A cultivar Bluebelle mostrou maior teor de potássio nos dois sistemas de cultivo, maior teor de cálcio no sistema semihidropônico e maior teor de magnésio no sistema convencional. As cultivares analisadas não se diferenciaram para os teores de cobre e ferro.

$\mathrm{O}$ nitrogênio apresentou-se insuficiente no sistema convencional para as duas cultivares. No sistema semi-hidropônico, mostrou-se normal para a cultivar Woodard e abaixo do normal para a cultivar
Bluebelle; no entanto, as plantas não apresentaram sintomas de deficiência. A quantidade normal de $\mathrm{N}$ no mirtileiro varia de 18,0 a 21,0 $\mathrm{g} \mathrm{kg}^{-1}$ (Sociedade Brasileira de Ciência do Solo. Comissão de Química e Fertilidade do Solo, 2004). O aumento do teor de $\mathrm{N}$ favorece o crescimento vegetativo, porém o seu excesso pode não ser benéfico para algumas culturas. Neste trabalho, a maior disponibilidade de $\mathrm{N}$ no sistema semi-hidropônico pode ter proporcionado a maior massa fresca e seca da parte aérea das cultivares analisadas.

Quando comparados com os teores normais de fósforo (1,2 a 4,0 $\mathrm{g} \mathrm{kg}^{-1}$ ) descritos no Manual de adubação e calagem para os Estados do Rio Grande do Sul e Santa Catarina, os teores desse nutriente nas duas cultivares analisadas se encontravam abaixo do normal no sistema convencional, e normal no sistema semi-hidropônico.

O potássio foi considerado acima do normal ou excessivo para todos os tratamentos. Os teores normais desse nutriente são de 3,5 a $6,5 \mathrm{~g} \mathrm{~kg}^{-1}$. O excesso desse nutriente poderia ter causado a deficiência de cálcio que também foi observada em todos os tratamentos.

Os teores de cálcio foram abaixo do normal ou insuficientes para todos os tratamentos. Apesar disso, as plantas não apresentavam sintomas visuais de deficiência desse elemento, como, por exemplo, necrose nos ápices radiculares ou nas folhas jovens. A quantidade normal de Ca para o mirtileiro varia de 4,0 a $8,0 \mathrm{~g} \mathrm{~kg}^{-1}$ (Sociedade Brasileira de Ciência do Solo. Comissão de Química e Fertilidade do Solo, 2004).

O magnésio apresentou-se normal (1,2 a 2,5 $\mathrm{g} \mathrm{kg}^{-1}$ ) para a cultivar Woodard nos dois sistemas de cultivo e acima do normal para a cultivar Bluebelle no sistema convencional.

A disponibilidade dos micronutrientes tem relação com o pH do solo ou substrato, e quando este é alto, os micronutrientes ficam indisponíveis. Neste estudo, o pH da solução nutritiva foi regulado para 5,0, que é considerado o $\mathrm{pH}$ ideal para a cultura do mirtileiro.

Os teores de cobre foram abaixo do normal ou insuficientes para todos os tratamentos. Entretanto, não foram observados sintomas visuais de deficiência desse nutriente. Os teores considerados normais de $\mathrm{Cu}$ para a cultura do mirtileiro é de 11 a $20 \mathrm{mg} \mathrm{kg}^{-1}$.

A concentração de zinco das cultivares de mirtileiro testadas nos dois sistemas de cultivo está dentro da normalidade (15 a $30 \mathrm{mg} \mathrm{kg}^{-1}$ ).

Os teores de ferro apresentaram-se acima do normal em todos os tratamentos. Porém as mudas não apresentavam sintomas de excesso deste elemento. Os teores normais de Fe em mirtileiro são de 81 a $199 \mathrm{mg} \mathrm{kg}^{-1}$. 
Em comparação com os teores normais de manganês (51 a $349 \mathrm{mg} \mathrm{kg}^{-1}$ ), o Mn mostrou-se abaixo do normal ou insuficiente no sistema convencional para as duas cultivares testadas, e normal no sistema semi-hidropônico.
A quantidade de nutrientes absorvida pelas plantas é função das condições edafoclimáticas e das características genéticas da planta. Dentre os fatores edafoclimáticos, a disponibilidade de nutrientes e a umidade do solo têm sido considerados como os principais responsáveis por alterações nos padrões de absorção de nutrientes.

A

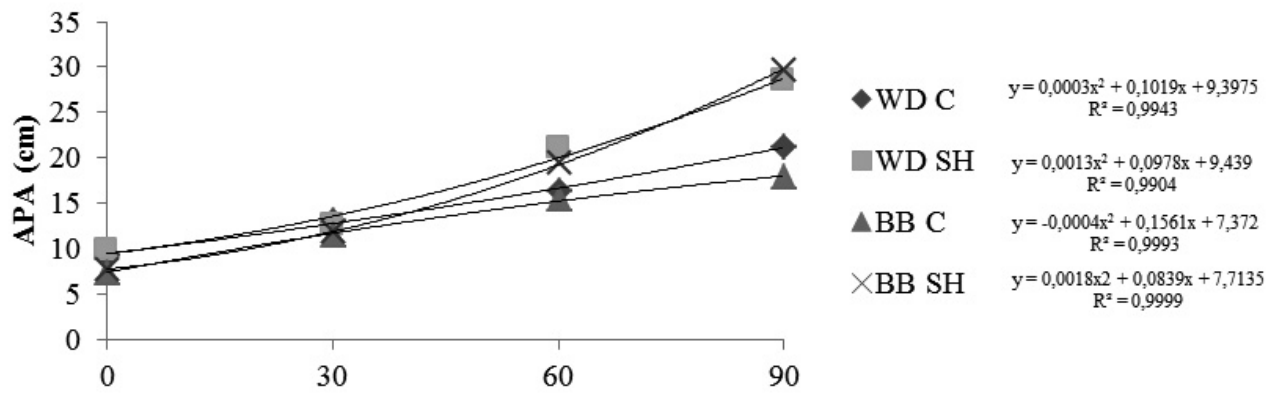

Tempo de cultivo (dias)

B

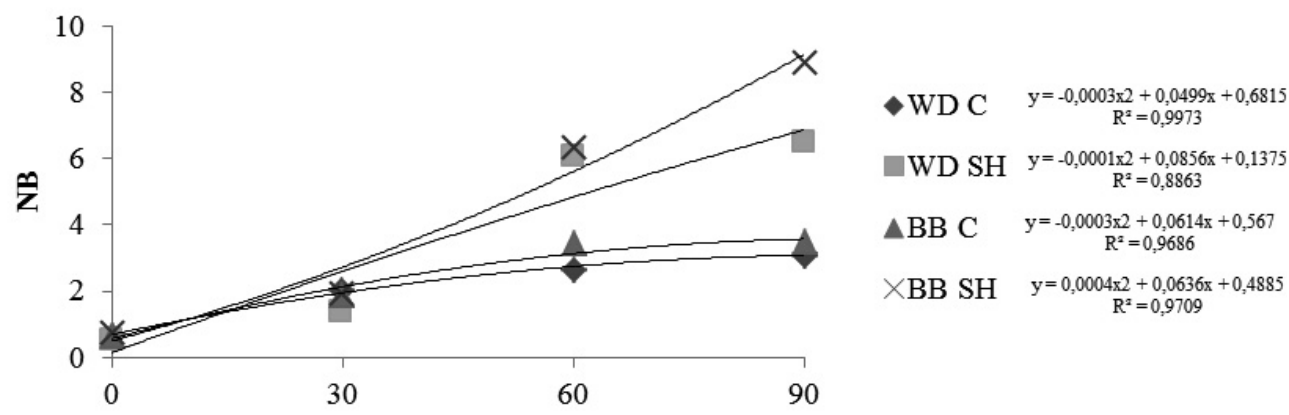

Tempo de cultivo (dias)

FIGURA 1 - Altura da parte aérea (APA) e número de brotações (NB) da cultivar Woodard em sistema convencional (WD C) e semi-hidropônico (WD SH) e da cultivar Bluebelle em sistema convencional (BB C) e semi-hidropônico (BB SH), em relação ao tempo de cultivo. 


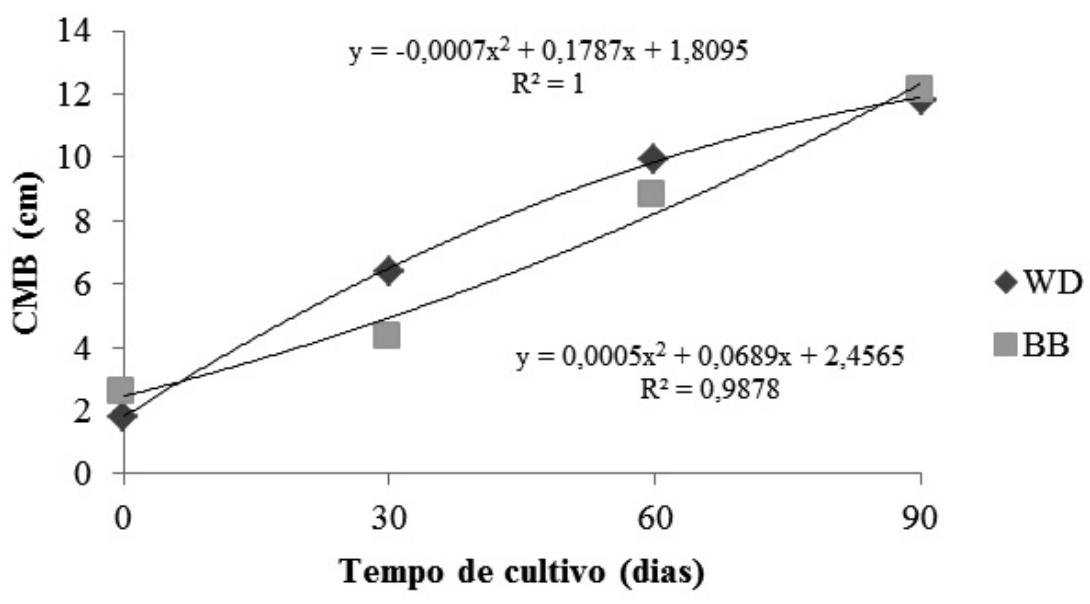

FIGURA 2 - Comprimento médio de brotações (CMB) das cultivares de mirtileiro Woodard (WD) e Bluebelle (BB) em relação ao tempo de cultivo.

TABELA 1 - Rendimento em microestacas (RM), massa fresca da parte aérea (MFPA), massa fresca radicular (MFR) e massa seca radicular (MSR) de mudas de mirtileiro das cultivares Woodard (WD) e Bluebelle (BB), em sistema convencional (C) e semi-hidropônico (SH).

\begin{tabular}{ccccccccc}
\hline & \multicolumn{2}{c}{ RM } & \multicolumn{2}{c}{ MFPA (g) } & \multicolumn{2}{c}{ MFR (g) } & \multicolumn{2}{c}{ MSR (g) } \\
\hline Sistema & WD & BB & WD & BB & WD & BB & WD & BB \\
SH & $26,39 \mathrm{Ab}$ & $33,64 \mathrm{Aa}$ & $222,04 \mathrm{Aa}$ & $233,53 \mathrm{Aa}$ & $70,84 \mathrm{Aa}$ & $30,14 \mathrm{Ab}$ & $23,04 \mathrm{Aa}$ & $13,62 \mathrm{Ab}$ \\
$\mathrm{C}$ & $13,47 \mathrm{Ba}$ & $14,71 \mathrm{Ba}$ & $51,77 \mathrm{Ba}$ & $43,79 \mathrm{Ba}$ & $21,00 \mathrm{Ba}$ & $20,81 \mathrm{Aa}$ & $5,93 \mathrm{Ba}$ & $5,90 \mathrm{Ba}$ \\
\hline
\end{tabular}

* Letras minúsculas na linha e maiúsculas na coluna não diferem estatisticamente entre si, pelo teste de Tukey, ao nível de 5\% de significância.

TABELA 2 - Teores de macronutrientes de folhas de mirtileiro das cultivares Woodard (WD) e Bluebelle (BB) mantidas em sistemas de cultivo convencional (C) e semi-hidropônico (SH).

\begin{tabular}{|c|c|c|c|c|c|c|c|c|c|c|}
\hline \multirow[b]{2}{*}{ Sistema } & \multicolumn{2}{|c|}{$\mathrm{N}\left(\mathrm{g} \mathrm{Kg}^{-1}\right)$} & \multicolumn{2}{|c|}{$\mathrm{P}\left(\mathrm{g} \mathrm{Kg}^{-1}\right)$} & \multicolumn{2}{|c|}{$\mathrm{K}\left(\mathrm{g} \mathrm{Kg}^{-1}\right)$} & \multicolumn{2}{|c|}{$\mathrm{Ca}\left(\mathrm{g} \mathrm{Kg}^{-1}\right)$} & \multicolumn{2}{|c|}{$\operatorname{Mg}\left(\mathrm{g} \mathrm{Kg}^{-1}\right)$} \\
\hline & WD & $\mathrm{BB}$ & WD & $\mathrm{BB}$ & WD & $\mathrm{BB}$ & WD & $\mathrm{BB}$ & WD & $\mathrm{BB}$ \\
\hline $\mathrm{SH}$ & $19,07 \mathrm{Aa}$ & $17,27 \mathrm{Ab}$ & 1,73 Aa & $1,35 \mathrm{Aa}$ & $9,39 \mathrm{Ab}$ & $2,46 \mathrm{Aa}$ & $3,08 \mathrm{Ab}$ & $3,47 \mathrm{Aa}$ & 2,70 Aa & 2,77 Aa \\
\hline $\mathrm{C}$ & $11,40 \mathrm{Ba}$ & $9,89 \mathrm{Bb}$ & $0,90 \mathrm{Ba}$ & $0,86 \mathrm{Ba}$ & $6,77 \mathrm{Bb}$ &, $77 \mathrm{Ba}$ & $2,64 \mathrm{Ba}$ & $2,68 \mathrm{Ba}$ & $1,41 \mathrm{Bb}$ & $1,68 \mathrm{Ba}$ \\
\hline
\end{tabular}

*Letras minúsculas na linha e maiúsculas na coluna não diferem estatisticamente entre si, pelo teste de Tukey, ao nível de 5\% de significância.

TABELA 3 - Teores de micronutrientes de folhas de mirtileiro das cultivares Woodard (WD) e Bluebelle (BB) mantidas em sistemas de cultivo convencional (C) e semi-hidropônico (SH).

\begin{tabular}{|c|c|c|c|c|c|c|c|c|}
\hline & \multicolumn{2}{|c|}{$\mathrm{Cu}\left(\mathrm{mg} \mathrm{Kg}^{-1}\right)$} & \multicolumn{2}{|c|}{$\mathrm{Zn}\left(\mathrm{mg} \mathrm{Kg}^{-1}\right)$} & \multicolumn{2}{|c|}{$\mathrm{Fe}\left(\mathrm{mg} \mathrm{Kg}^{-1}\right)$} & \multicolumn{2}{|c|}{$\operatorname{Mn}\left(\mathrm{mg} \mathrm{Kg}^{-1}\right)$} \\
\hline Sistema & WD & BB & WD & BB & WD & BB & WD & $\mathrm{BB}$ \\
\hline SH & $4,15 \mathrm{Aa}$ & 5,94 Aa & $24,63 \mathrm{Aa}$ & $20,80 \mathrm{Aa}$ & $326,39 \mathrm{Aa}$ & $337,82 \mathrm{Aa}$ & $248,06 \mathrm{Aa}$ & $129,57 \mathrm{Ab}$ \\
\hline $\mathrm{C}$ & $3,35 \mathrm{Ba}$ & $3,87 \mathrm{Aa}$ & $24,39 \mathrm{Aa}$ & $17,45 \mathrm{Ab}$ & $213,36 \mathrm{Aa}$ & $271,78 \mathrm{Aa}$ & $5,84 \mathrm{Ba}$ & $27,08 \mathrm{Ba}$ \\
\hline
\end{tabular}

Letras minúsculas na linha e maiúsculas na coluna não diferem estatisticamente entre si, pelo teste de Tukey, ao nível de 5\% de significância. 


\section{CONCLUSÃO}

O sistema semi-hidropônico mostra-se eficiente para o crescimento de mudas de mirtileiro, sendo significativamente superior ao método convencional de cultivo.Aos 60 dias de cultivo em sistema semi-hidropônico, pode haver plantas aptas para a coleta de microestacas..As mudas cultivadas no sistema semi-hidropônico apresentam teores de nutrientes mais próximos aos considerados normais para a cultura do mirtileiro, em comparação ao sistema convencional.

\section{REFERÊNCIAS}

ANTUNES, L.E.C.; GONÇALVES, E.D.; TREVISAN, R.; RISTOW, N.C. Sistema de produção do mirtilo. Pelotas: Embrapa Clima Temperado, 2007 (Sistemas de Produção, 8). Disponível em: $<$ http://sistemasdeproducao.cnptia.embrapa.br/ FontesHTML/Mirtilo/SistemaProducaoMirtilo/ propagacao.htm> Acesso em: 22 nov. 2010.

CHRISTOFIDIS, D. Irrigação, a fronteira hídrica na produção de alimentos: Irrigação e tecnologia moderna. Brasília: ABID, 2002. n.54, p.46-55.

CUNHA, A.C.M.; WENDLING, I.; JUNIOR, L.S. Miniestaquia em sistema de hidroponia e em tubetes de corticeira-do-mato. Ciência Florestal, Santa Maria, v. 18, n. 1, p. 85-92, 2008.

FREIRE. C.J. da S. Nutrição e adubação para mirtilo. In: RASEIRA, M. do C.; ANTUNES, L.E.C. (Ed.). A cultura do mirtilo. Pelotas: Embrapa Clima Temperado, 2006. p. 60-74. (Sistemas de produção, 8).

FURLANI, P.R. Cultivo de frutas e hortaliças em ambiente protegido. Fortaleza: Instituto Frutal, 2009. 37p.

MACHADO, A.A.; CONCEIÇÃO, A.R. Sistema de análise estatística para windows. WinStat. Versão 2.0. Pelotas: UFPel, 2003.
PAYNE, T. J. Formulating with Blueberries for Health. Cereal Foods World, Mineapolis, v. 50, n. 5, p. 262-264, 2005.

RISTOW, N.C.; ANTUNES, L.E.C.; SCHUCH, M.W.; TREVISAN, R. Crescimento de plantas de mirtilo a partir de mudas micropropagadas. Revista Brasileira de Fruticultura, Jaboticabal, v. 31, n. 1, 2009.

SANTOS, A.M. dos; RASEIRA, M. do C.B. A cultura do mirtilo. Pelotas: Embrapa Clima Temperado, 2002. 23 p. (Documentos, 96)

SCHUCH, M.W.; DAMIANI, C.R.; SILVA, L.C. da; ERIG, A.C. Micropropagação como técnica de rejuvenescimento em mirtilo (Vaccinium ashei Reade) cultivar Climax. Ciência e Agrotecnologia, Lavras, v. 32, n. 3, p. 814-820, 2008.

SCHUCH, M.W.; PEIL, R.M.N. Soilless cultivation systems: A new approach in fruit plants propagation in the south of Brazil. In: INTERNATIONAL SYMPOSIUM ON ADVANCED TECHNOLOGIES AND MANAGEMENT TOWARDS SUSTAINABLE GREENHOUSE ECOSYSTEMS-GREEN SYSZOLL, 2011. No prelo

SOCIEDADE BRASILEIRA DE CIÊNCIA DO SOLO. COMISSÃO DE QUÍMICA E FERTILIDADE DO SOLO. Manual de adubação e calagem para os Estados do Rio Grande do Sul e de Santa Catarina. 10. ed. Porto Alegre, 2004. 400p.

SOUZA, J.L. de. Tipo de minijardim clonal e efeito do ácido indolbutírico na miniestaquia de Grevillea robusta A. Cunn. (Proteaceae). 2007. Dissertação (Mestrado) - Setor de Ciências Biológicas, Universidade Federal do Paraná, Curitiba, 2007.

TEDESCO, M. J.; GIANELLO, C.; BISSANI, C. A.; BOHNEN, H.; VOLKWEISS, S. J. Análises de solo, plantas e outros materiais. 2. ed. rev. e ampl. Porto Alegre: Departamento de Solos, UFRGS, 1995. 174p. 